\title{
Multi-RAT Aggregation through Spectrum Reallocation for Future Wireless Networks
}

\author{
Apostolos Galanopoulos*, Fotis Foukalas* • \\ Theodoros Tsiftsis ${ }^{+}$ \\ *Industrial Systems Institute, \\ Athena Research and Innovation Center, \\ Patras, Greece \\ + School of Electrical and Information \\ Engineering, Jinan University (Zhuhai \\ Campus), Zhuhai 519070, China
}

Received: date / Accepted: date

\begin{abstract}
Next generation wireless networks are becoming the main focus of the industry by putting efforts to launch beyond $4 \mathrm{G}$ (i.e. $5 \mathrm{G}$ ) communication systems by 2020 . Towards the $5 \mathrm{G}$-system vision, the efficient spectrum aggregation by integrating multiple radio access technologies (multi-RAT) is one of the enablers to achieve the highest data rates. To this end, a multi-RAT aggregation is envisioned that can be provided using the spectrum reallocation technique. Spectrum reallocation among multi-RATs can provide spectrum opportunities for aggregation and, thus, the overall spectrum utilization and network capacity increase. Maintaining an optimum quality of experience (QoE) for users of different RATs in such an extremely complex network environment can be facilitated by such a multi-RAT aggregation (spectrum aggregation from different $R A T s$ ), through spectrum reallocation. To this end, both network and functional architectures are specified and spectrum assignment solutions are proposed in this article. The goal is to efficiently increase the data rates supporting a required QoE for all users.
\end{abstract}

Keywords spectrum rallocation · spectrum refarming $\cdot$ multi-RAT $\cdot$ carrier aggregation $\cdot$ joint coordination.

A. Galanopoulos · F. Foukalas

I.S.I. - Industrial Systems Institute

Platani, PATRAS, Greece, 26504

Cell.: +30-6977562678

Fax: +30 2610910.303

E-mail: foukalas@ieee.org 


\section{Introduction}

Next generation (5G) cellular networks heavily rely on the Carrier Aggregation (CA) technique which can greatly improve data rates [1]. LTE-A utilizes this technique in recent releases but the problem is that there is not enough spectrum reserved for LTE-A to accommodate the increasing number of subscribers with LTE-A Radio Access Technology (RAT) devices. Since the number of LTE-A users is continuously rising, due to the migration of legacy RAT (e.g. GSM, HSPA) subscribers to LTE-A, the bandwidth requirements of LTE-A networks to support a minimum QoE level to their users also increase. Furthermore, since less users access the legacy RATs, a large amount of spectrum dedicated to them is left underutilized and although next generation solutions are slowly taking shape, it is evident that a single (new) radio technology can not guarantee the efficient reuse of spectrum [2][3].

A promising way of providing more spectrum to LTE-A systems is by coordinating the exploitation of spectrum assigned to multiple legacy RATs and reallocate it to LTE-A systems where the need is greater. 3GPP has issued a study about the joint coordination of m-RAT systems [4], in order to describe the cases where coordination mechanisms can be applied between different RATs in order to efficiently exploit a spectrum band that is gradually abandoned by a legacy RAT, creating spectrum holes, and assign the surplus spectrum to the LTE-A. This additional spectrum to the LTE-A system can be utilized using CA for dynamic spectrum expansion. Such proposition is also presented in [5], where solutions for specific scenarios between coordinating RATs are being proposed. Towards future wireless networks, SR is able to provide aggregation of spectrum that belongs to different RATs, named multi-RAT aggregation, addressing the challenge of spectrum reuse [6]. Spectrum re-farming is considered as a common practice to statically reallocate the spectrum without changing the available spectrum size though[7]. Due to static nature of spectrum re-farming, policy makers and regulators would prefer a type of spectrum sharing especially to access spectrum below $6 \mathrm{GHz}$ [8] [9]. Such a problem is more evident in case of multi-RAT use cases. To this end, the dynamic spectrum refarming, i.e. spectrum reallocation, is considered as the solution to deal with the efficient spectrum utilization among different RAT[10].

Regarding the Spectrum Reallocation (SR), there are some significant works to mention. In particular, in [11], a dynamic spectrum management solution is provided for a m-RAT system where each RAT is assigned with a chunk of shared spectrum proportional to the its traffic demands. Simulation results show an improved performance compared to static spectrum assignment strategies. The authors in [12] present a survey of existing bandwidth aggregation techniques. In [13], it is shown how an under-utilized GSM band can be reallocated to LTE, while also considering the support of GSM devices with less spectrum, by jointly allocating resources with respect to inter-RAT 
interference mitigation. The authors of [14] propose a dynamic SR scheme between GSM and LTE networks. Simulation results show that even under heavy GSM load, the potential for LTE capacity gains is significant. The work in [15] studies the energy efficient joint resource allocation of two OFDMA based RATs. The solution is based in two stages with the first one providing subcarrier allocation assuming equal power allocation, and the second one deciding the actual power allocation in order to maximize the energy efficiency. Energy efficiency is also considered in [16] were a dynamic solution for splitting video traffic between $\mathrm{WiFi}$ and cellular interfaces is presented. The proposed algorithm, based on Lyapunov optimization, demonstrates how users' QoE is affected by the RAT they use. Evidently, proper and dynamic adjustment of the allocated spectrum is necessary for satisfying the users' constantly changing requirements. In [17], [18] the joint operation of OFDMA and CDMA systems is analyzed to provide a resource allocation solution that guarantees a minimum SINR (signal-to-interference-noise-ratio) value for the CDMA system while trying to maximize the throughput performance of the OFDMA system. M-RAT is also studied using software defined networking in [19] for mobility reconfiguration though. In [20], the authors also studied a multi-RAT scenario focuding on HetNets deployments from QoS point of view. In the proposed algorithm, users are assigned to the most suitable RAT towards satisfying their QoS requirements. In [21], a m-RAT joint resource allocation method is proposed based on a proportional fairness scheduler that considers the traffic demand and channel state information (CSI) of all users of each RAT. On top of that, a unified Radio Access Network (RAN) architecture is presented in order to coordinate the joint operation of the m-RAT system. Simulations were carried out for a co-deployed HSPA/LTE-A system and compare the throughput performance of both systems under dynamic spectrum assignment with disjoint scheduling and the proposed joint resource allocation. The results indicate increased overall system performance under all tested scenarios. In addition, joint resource management techniques in m-RAT systems that rely on reinforcement learning have been studied in [22]. The authors consider a LTE-UMTS collocated deployment where the reinforcement learning agent decides the serving RAT for each user based on the reward function values they obtain for each RAT.

In this work, we propose a multi-RAT SR framework that can provide spectrum aggregation expanding the concept of carrier aggregation (CA) to a larger scope. We first provide an overview of SR as defined within 3GPP Rel.13 and next, we present our proposal for multi-RAT SR, where the network architecture and the functional architecture are specified. A multi-RAT controller is responsible for the coordination of the dynamic spectrum assignment and reallocation that is driven by the QoE module. Finally, the multi-RAT spectrum assignment and reallocation solutions are devised and explained providing specific results to indicate the achievable performance. The proposed solution can facilitate the dynamic spectrum aggregation among different RATs. 
The remainder of this paper is organized as follows. Sec.II presents and overview of SR introduced within 3GPP. In Sec.III, two proposals for m-RAT SR are made and then, analyzed in Sec.IV followed by simulation results comparing the performance of the proposed solutions. Finally, Sec.V concludes this paper.

\section{Multi-RAT Spectrum Reallocation Overview}

3GPP defines Spectrum ReFarming (SRF) and Spectrum Reallocation (SR) in [4]. In particular, the $\mathrm{SR}$ is the term used to describe the procedure of reallocating spectrum that is initially assigned to some legacy RAT, to the spectrum demanding LTE-A technology. This procedure can be applied in several cases with respect to the frequency at which reallocation decisions are made depending on the traffic load of legacy RAT and LTE-A networks. The cases of SR considered are the following [4]:

Dynamic. SR occurs every few seconds/minutes. It can guarantee maximum efficiency and utilization of spectrum resources for all co-deployed RATs.

Semi-static. SR occurs every few hours/days. Several preconfigured plans can be defined to alter resource partitioning between RATs in a fixed way according to current traffic conditions and requirements.

Static. SR occurs every few months/years. This is the case of the so called SRF, where a legacy RAT band is reallocated permanently to LTE for exclusive exploitation [17], where obviously SRF is a sub-category of SR. The solutions considered so far, only concern static and semi-static SR due to the fact that the required mechanisms to exchange data between RATs are simple. However, the gains of Dynamic SR (DSR), where spectrum and resource allocation decisions are made more frequently are expected to overcome the increased architectural complexity it entails.

A generic description of the SR is conceptualized in Fig. 1. Fig. 1(a) depicts the original (i.e. according to the regulations) spectrum assignment, where the legacy RAT is assigned with large spectrum that is underutilized as a whole. Under the SR concept, this spectrum chunk is available in a shared fashion and can be assigned to any RAT (legacy or LTE-A) depending on the traffic requirements. In Fig. 1(b), the Static SR (i.e. SRF) is illustrated, where the shared band is exclusively reallocated to LTE-A. The next case is the DSR depicted in Fig. 1(c), where the assignment of the shared band between the two RATs is provided in a dynamic fashion according to the traffic load of each RAT, aiming to satisfy both legacy and LTE-A users. As network traffic conditions change, $S_{1}$ spectrum chunk is dynamically altered to shape the two RATs overall spectrum assignment, i.e. $B W_{1}$ and $B W_{2}$. Efficiency is considered in terms of assigning spectrum to users experiencing better channel conditions or need extra bandwidth to satisfy their QoS requirements. Further, in case of carrier aggregation $(\mathrm{CA})$ enabled technologies, e.g. $2.5 \mathrm{G} / 3 \mathrm{G} / 4 \mathrm{G}$, the shared band can be divided into a number of component carriers (CCs) (Fig. 1(d)), part of it $\left(S_{1}\right)$ is assigned to the legacy RAT in parallel to the originally 


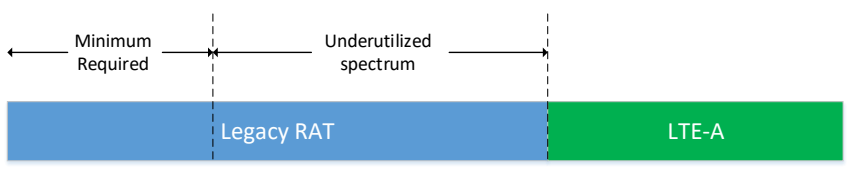

(a)

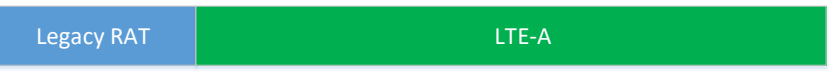

(b)

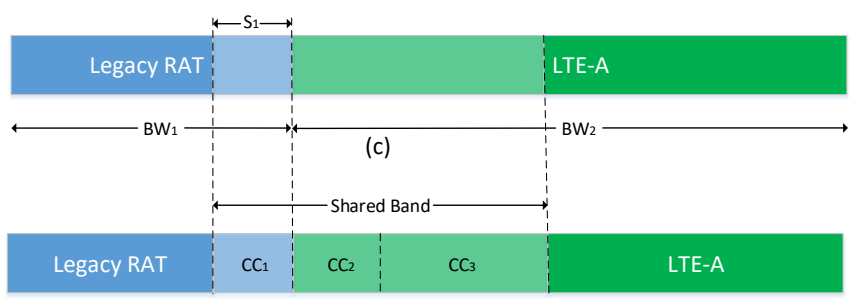

(d)

Fig. 1 Spectrum Reallocation within the 3GPP framework.

assigned bandwidth, and the rest of the spectrum is exploited by LTE-A. CA can provide to the DSR solution an additional flexibility through agile spectrum management and allocation mechanisms.

Further, the key idea about m-RAT joint coordination is to define under which conditions different RATs deployed in the same geographical area can communicate in order to perform some sort of spectrum assignment and resource allocation, by making as few modifications to the system architecture as possible. Special coordination functions should be implemented for this joint resource allocation at each RAT, or through a coordination point that collects information mainly about the load and the average data rate of the users of each RAT [5]. About the available spectrum, the specified scenarios define that the legacy RAT has a minimum required bandwidth to satisfy a certain data rate need and the rest of it is pooled for the coordination mechanism to decide the allocation.

\section{Multi-RAT Spectrum Reallocation Architecture}

This section illustrates how multi-RAT SR can be supported by a particular network and functional architecture. 


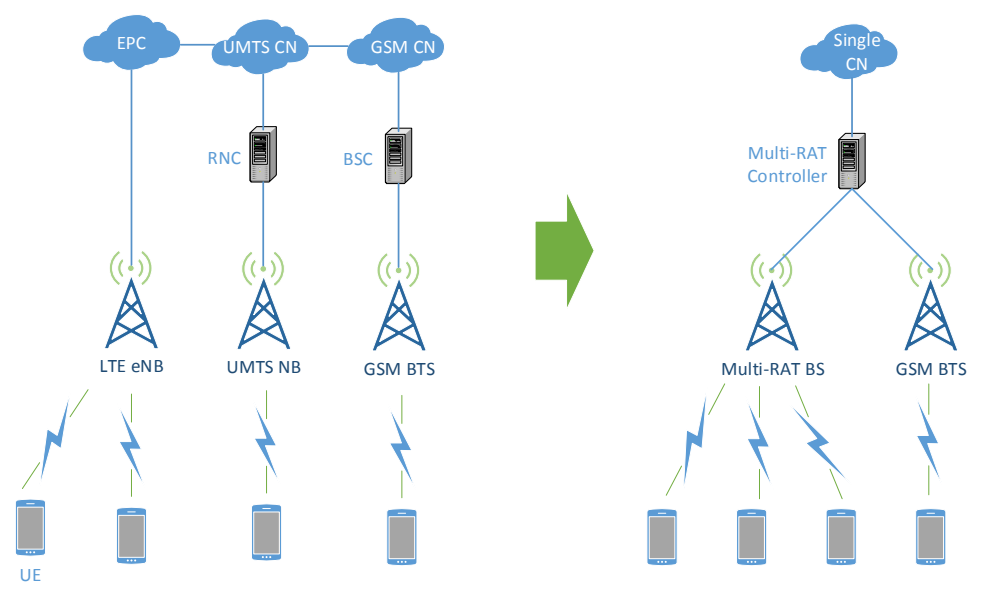

Fig. 2 Multi-RAT network architecture.

\subsection{Network Architecture}

First, we show how the architecture can be applied to an LTE-Advanced (LTEA) system in order to guarantee backward compatibility. Conventionally, LTEA systems operate in licensed bands and therefore do not employ any opportunistic access; this necessitates such architecture as the one introduced below. The implementation of the coordination functions will definitely impact network architecture, since two or more RATs that are often not collocated need to communicate with each other in order to mutually assign spectrum. To this end, the introduction of a unified Multi-RAT Controller (m-RC) is proposed $[5,23]$.

The m-RC manages a number of Base Stations (BSs) of different RATs and additionally to the spectrum assignment mechanism implements other legacy RAT functionalities such as scheduling, mobility management and interference coordination between neighbouring cells. An illustration of the proposed network architecture is given in Fig. 2, where different RATs are possibly unified in a Multi-RAT BS and communicate with the Core Network $(\mathrm{CN})$ through a single $\mathrm{m}-\mathrm{RC}^{1}$. In multi-RAT $\mathrm{SR}$, the $\mathrm{m}-\mathrm{RC}$ directly communicates with all BSs of different RATs in order to provide the spectrum assignment and resource allocation decisions. This more likely will introduce some delay restrictions

1 The LTE-A architecture does not incorporate any controller, and the aforementioned functionalities are im-plemented in a distributed way among neighbouring eNBs. 
for LTE-A, if scheduling is handled jointly for all RATs by the controller [17, 24], since LTE-A scheduling is handled by the eNBs. For legacy RATs such as UMTS and GSM, the system architecture remains the same, where the m-RC integrates all the functions included in each RATs controller, namely Radio Network Controller (RNC) and Base Station Controller (BSC) already known and specified in UMTS and GSM, respectively.

\subsection{Functional Architecture}

The m-RC integrates the legacy RATs functionalities (previously defined in the RNC and the BSC) as well as new joint Radio Resource Management (RRM) functions towards supporting SR (Fig. 3). A Measurement Collector module is required so that a joint RRM function can utilize Channel State Information (CSI) from User Equipments (UEs) of multi-RATs. Such information is forwarded by the associated BSs using each RATs specific interface. The unified Interference Coordination Manager is a special entity since interRAT interference problems can rise (especially between GSM and LTE-A) in $\mathrm{SR}$ scenarios [5,17]. In order to perform fair and efficient RRM, a QoE module is also embedded into the m-RC. It aims to handle QoE requirements for each UE associated with a BS, and match them to QoS requirements, e.g. throughput, delay, etc. [25]. This is typically accomplished by utilizing the Mean Opinion Score (MOS) for specific applications [26]. Further, some machine learning (ML) can be also used to correlate QoE with QoS requiremens [27]. ML is considered as a powerful too, where the training of different QoE prediction models can lead to good correlation [28]. However, we use the MOS type of matching as more practical in case of our multi-RAT spertrum reallocation solution.

The interfaces between the m-RC and the BSs are specific for each RAT so that the design is back-ward compatible with legacy RAT BSs. For the UMTS, the Iub interface connects a NB to the RNC functions module to support UMTS functionality. For the GSM, the respective interface used to connect BTSs with the BSC functions module is the Abis interface [29]. Since LTE-A does not require a central controller, the eNBs are directly connected to the CN. The X2 interface that is typically used to interconnect eNBs can also be used for Connection with the $\mathrm{m}-\mathrm{RC}$. This is possible because the $\mathrm{X} 2$ interface supports information exchange between load management functions of different eNBs and can be helpful to the Measurement Collector of the mRC. The measurements can load information from the associated UEs and forward them to the Joint RRM. The Interference Coordinator Manager can also be used by the joint RRM function, in case that the involved RATs are sensitive to inter-RAT interference and need to be assigned with spectrum with a minimum spacing to avoid interference. Finally, the QoE module maps the QoE requirements of each UEs to QoS requirements, so that the RRM 


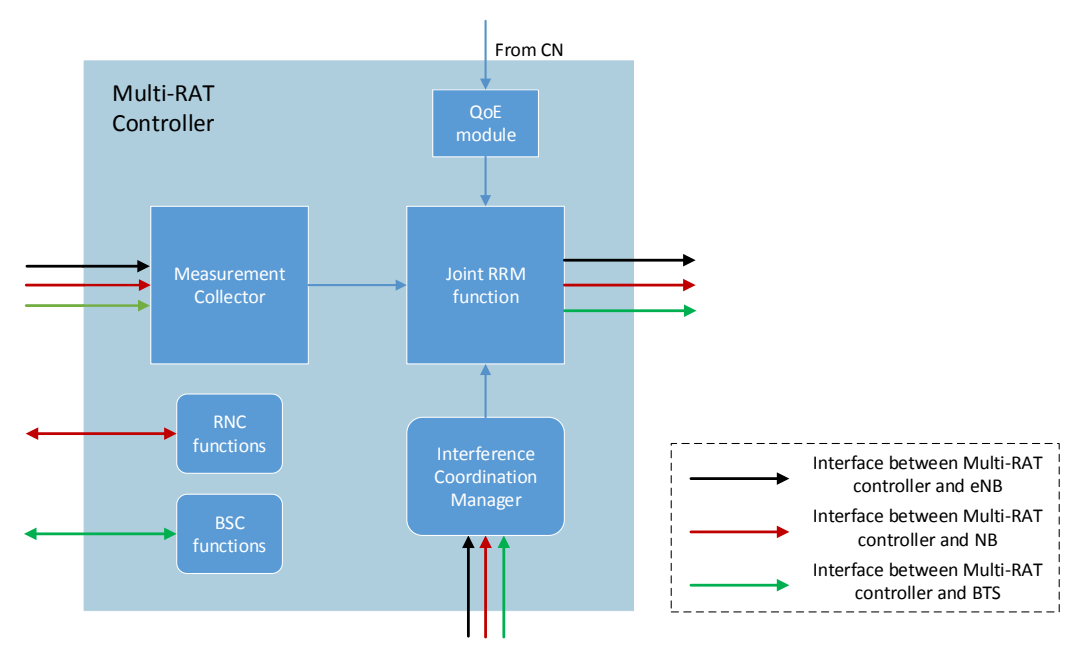

Fig. 3 Multi-RAT Controller (m-RC) functional diagram.

function assigns spectrum accordingly. After spectrum assignment, the results are forwarded to the BSs through the appropriate interfaces.

For the remainder of this document we consider two co-deployed RATs (a legacy RAT and LTE-A) each one operating at its own frequency band. Under the m-RAT joint coordination scenario, we assume that the legacy RAT band is defined to be shared between the two RATs in order to develop coordination mechanisms for efficient DSR. Spectrum assignment and resource allocation of the m-RAT system [5] under the shared band assumption can lead to creating new ways of employing $\mathrm{CA}$ as described below.

\section{Multi-RAT Aggregation through Spectrum Reallocation}

This section provides spectrum reallocation solutions enabling the multi-RAT (spectrum) aggregation.

\subsection{System Setup}

We assume a continuous bandwidth of $50 \mathrm{MHz}$ like in Fig. 1(b). As an example it is noted that such wide bands exist, e.g. bands $1,3,7$ are even wider at 60 , 75 and $70 \mathrm{MHz}$ respectively. Suppose that originally the legacy RAT owned 30 $\mathrm{MHz}$ of bandwidth and LTE-A the next $20 \mathrm{MHz}$. Due to the users migration to 
Table 1 Simulation Parameters

\begin{tabular}{|l||l|l|}
\hline Parameter & HSPA & \multicolumn{2}{|c|}{ LTE-A } \\
\hline Duration & \multicolumn{2}{|c|}{ E00 frames } \\
\hline Channel & \multicolumn{2}{|c|}{ EPA } \\
\hline Modulation & \multicolumn{2}{|c|}{ Adaptive } \\
\hline Supported Bandwidths (MHz) & 5,10 & $1.4,3,5,10,15,20$ \\
\hline Scheduler & Round Robin & Proportional Fair \\
\hline Tx Scheme/MIMO & SISO & Diversity (2x2) \\
\hline Spreading Factor & 16 codes & - \\
\hline Chip Rate & $3.84,7.68 \mathrm{Mcps}$ & - \\
\hline Subcarrier Spacing & - & $15 \mathrm{KHz}$ \\
\hline Average Data Rate Threshold & $800 \mathrm{kbps}$ & No threshold \\
\hline
\end{tabular}

LTE-A, the minimum required bandwidth for the legacy RAT has minimized to $10 \mathrm{MHz}$. The next $20 \mathrm{MHz}$ of spectrum that were previously assigned to the legacy RAT, are now shared with LTE-A, and finally the remaining 20 $\mathrm{MHz}$ are still assigned to LTE-A only. The entire bandwidth can be split into a number of CCs that consist a set $\mathcal{K}$. For the rest of the analysis, we define the legacy RAT as RAT-1 to be HSPA and LTE-A as RAT-2, as well as the sets $\mathcal{N}$ and $\mathcal{M}$ to be the sets of users of RAT-1 and RAT-2 respectively. The development of the simulation and HSPA/LTE processing chains was developed using Matlab. A summary of the simulation parameters used to acquire the results of the proposed solutions is presented in Table 1.

\subsection{Spectrum Assignment}

Spectrum Assignment ( $\mathrm{SpA}$ ) requires a specific function that collects information about the demand of the legacy RAT in bandwidth, considering the transmission capabilities of each RAT (e.g. HSPA supports specific bandwidths per Component Carrier (CC) ) and assigning the shared spectrum band to each RAT accordingly. After SpA is completed each RAT allocates resources to the assigned users individually with no respect to the operation of the other RAT. Note that depending on the legacy RAT, neighboring spectrum blocks that belong to different RATs may face Inter RAT Interference (IRI) problems (e.g. GSM is known to heavily impact LTE-A while HSPA does not). In addition, CA is employed in LTE-A between the originally assigned CC and the one(s) that result from the application of the SpA function. In case of the legacy RAT is HSPA, we also gain the benefit of employing CA in both RATs. On the architectural changes required to enable SpA, the necessary function can be implemented on the m-RAT eNodeB and coordination between the scheduling modules of each RAT is easy to support. Note that in this case, the two RATs may not be co-located and they utilize a coordinator to deliver their load information. The coordinator in turn, is in charge of implementing the SpA function and inform each RAT on the resulted spectrum assignment [5]. 
The implementation of the $\mathrm{SpA}$ function requires the m-RAT eNodeB to collect CSI from the associated users of HSPA as well as their data rate demands in order to assign the shared spectrum. The procedure is driven by the bandwidth demands of RAT-1 and if the minimum required bandwidth is sufficient to cover a minimum average data rate threshold. The data rate requirements of RAT-1 users are defined as:

$$
R^{1}=\left\{R_{1}^{1}, R_{2}^{1}, \ldots, R_{i}^{1}, \ldots, R_{N}^{1}\right\}
$$

where $R_{i}^{1}$ is the data rate required by user $i$. The average data rate threshold $R_{t h}$ that HSPA has to satisfy is provided by averaging the users requested data rates of $R^{1}$. In addition, the CSI values obtained from users $i \in \mathcal{N}$ for CC $k \in \mathcal{K}$ is defined as:

$$
C S I_{k}^{1}=\left\{C S I_{k, 1}^{1}, C S I_{k, 2}^{1}, \ldots, C S I_{k, i}^{1}, \ldots, C S I_{k, N}^{1}\right\},
$$

and for users $j \in \mathcal{M}$ as:

$$
C S I_{k}^{2}=\left\{C S I_{k, 1}^{2}, C S I_{k, 2}^{2}, \ldots, C S I_{k, j}^{2}, \ldots, C S I_{k, M}^{2}\right\} .
$$

Based on the CSI information of RAT-1 and the average rate requirement based on $R^{1}$, a calculation is carried out on the required bandwidth of RAT-1, $B W^{1}$ :

$$
B W^{1}=f_{1}\left(R^{1}, C S I_{1}^{1}, \ldots, C S I_{K}^{1}\right)
$$

Function $f_{1}$ is the $\mathrm{SpA}$ function. If $B W^{1}$ is greater than the minimum required bandwidth, a portion $S^{1}$ of the shared band has to be temporarily assigned to RAT-1 so that $S^{1}+10 M H z=B W^{1} . S^{1}$ is formed in CCs according to the bandwidths supported by RAT 1 . In our case, the $20 \mathrm{MHz}$ wide $S^{1}$ can either be divided to 2 of $10-\mathrm{MHz}$ CCs or 4 of $5-\mathrm{MHz}$ CCs. The remaining spectrum from the shared band is assigned to RAT 2, i.e. LTE-A with the same restriction about division in CCs to apply here as well. Both RATs are notified about spectrum assignment decisions and perform Resource allocation separately in the resulted number of CCs in order to satisfy their users. The advantage of CA RATs rises from the capability to allocate resources from different CCs to a single UE allowing higher data rates.

The functionality of $f_{1}$ is briefly described in Algorithm 1. Basically, a calculation of the achievable average data rate is made based on the assigned to HSPA bandwidth. If this data rate does not reach the required $R_{t h}$, then another CC is assigned to HSPA and the average data rate is recalculated until it has reached the required threshold.

\subsection{Joint Spectrum Assignment and Resource Allocation}

With Spectrum Assignment and Resource Allocation (SpARA) the entire shared band is divided into a number of CCs, which are assigned to 


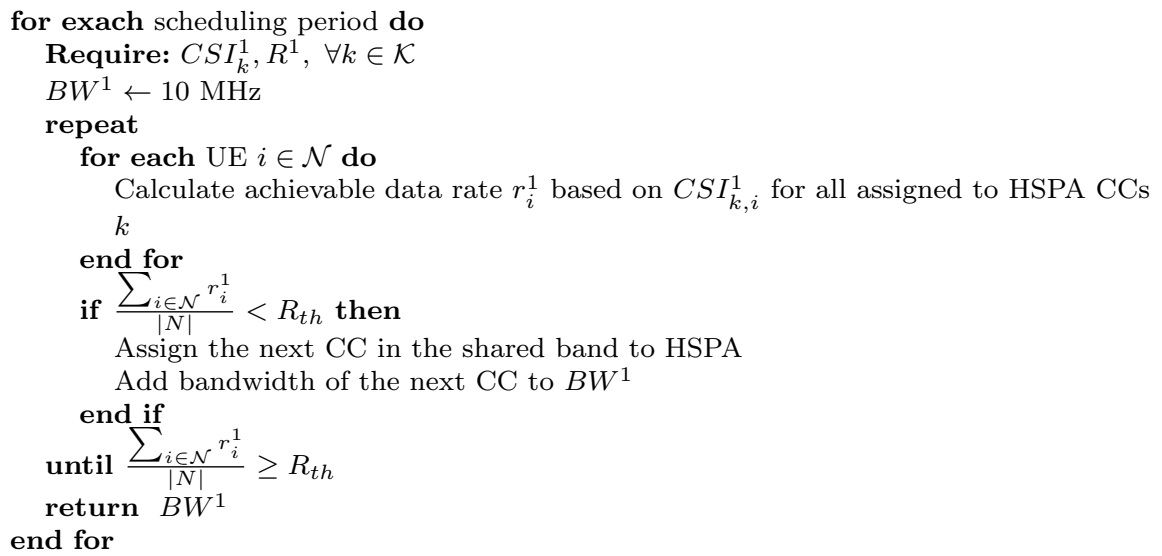

Algorithm 1: Spectrum Assignment function

either the legacy RAT or to LTE-A, and thus, utilize them for efficient multiCC CA. By this design however, a portion of the resulted CCs of the shared band are reserved for the support of the legacy RAT users. The exact CCs that are assigned to each RAT are a function of legacy RAT/LTE-A users' CSI and are chosen with respect to maximizing both systems' performance. This is a more promising technique compared to single SpA because through joint SpARA the spectrum reserved for the legacy RAT is exactly what is required, and the rest of it is exploited by LTE-A through CA. Moreover, through appropriate joint scheduling decisions each user is assigned with their best possible CCs regardless their employed RAT. The former legacy RAT CCs of the shared band that are reallocated to LTE-A and are aggregated with the original LTE-A CC can provide the so much required throughput performance boost to LTE-A users towards achieving 5G requirements. Since these CCs are probably underutilized by the legacy RAT, LTE-A can benefit from the resources of the legacy RAT band by allocating resources both for LTE-A and the legacy RAT in a common scheduler entity. Obviously, a joint SpARA function carries a heavier architectural modification load due to the integration of LTE-A/Legacy RAT Resource Allocation [21], but will also provide a more efficient spectrum utilization if designed carefully.

Compared to the single spectrum assignment function, the joint resource allocation is more complex due to the consolidation of the resource allocation modules to a single entity. Resource allocation is performed as normal, e.g. through the Proportional Fair (PF) scheduling to each RAT for the assigned bandwidths. As for the shared band, it is divided in a number of CCs in a way that they can all be utilized by either RAT. For the scenario depicted in Fig. 1, with a $20 \mathrm{MHz}$ shared band, this division can either be in $210-\mathrm{MHz}$ CCs or $45-\mathrm{MHz} \mathrm{CCs}$, as mentioned earlier, with both setups supported by HSPA and LTE-A. The aim is to assign each CC to the user that satisfies an optimal scheduling criterion independently of their RAT. To this end, a 
common scheduling algorithm that guarantees a level of fairness between users of different RATs needs to be employed (PF scheduling). Function $f_{2}$ that performs joint SpARA is expressed mathematically as:

$$
R A=f_{2}\left(C S I_{k}^{1}, C S I_{k}^{2}\right), \forall k \in \mathcal{K}
$$

The increased complexity of the joint Resource Allocation module is evident from the fact that requires a lot more information from users of both RATs and for a larger range of spectrum. The PF scheduling metric used is defined as a function of the instantaneous data rate experienced by user $i \in \mathcal{N} \cup \mathcal{M}$ in CC $k \in \mathcal{K}, R_{k, i}$ and its average experienced data rate $R_{i}^{a v g}$ [30]. $\mathrm{CC} k$ is then assigned to the user with the maximum scheduling metric for the specific CC. Formally it is:

$$
i_{k}^{*}=\arg \max _{i} \frac{R_{k, i}}{R_{i}^{a v g}},
$$

where $i_{k}^{*}$ is the user that is assigned with CC $k$. Note the proportional fairness characteristic of the metric that favors users with high instantaneous data rate and low average data rate. Since users experience different channel conditions for each CC, and thus $R_{k, i}$ differs for each $k$ value it is possible that the same user is assigned with multiple CCs at each scheduling period, consequently increasing the performance of both CA enabled RATs. The joint SpARA function $f_{2}$ operation is described in Algorithm 2. The scheduling period is the duration of a frame $(10 \mathrm{~ms})$, which is common for HSPA and LTE-A.

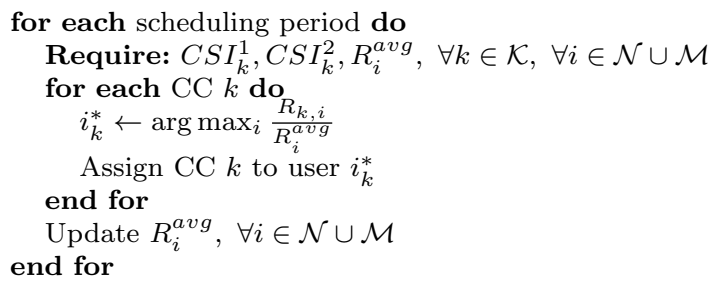

Algorithm 2: Joint Spectrum Assignment and Resource Allocation function

\subsection{QoE-driven spectrum reallocation}

An efficient SR requires knowledge of basic QoS requirements of the applications run by the UEs. Such knowledge is not straightforward available to the Multi-RAT networks and furthermore, the QoS characteristics are not always sufficient to ensure that the UEs experience high QoS. To this end, QoE rather than QoS requirements need to be considered but these requirements are subjective to the end users. There is a number of methods such as MOS 
Table 2 QoE to QoS requirements mapping for different type of services

\begin{tabular}{|l||l|l|l|l|}
\hline Service & $\begin{array}{l}\text { QoS Parame- } \\
\text { ter }\end{array}$ & MOS=3 & MOS=4 & MOS=5 \\
\hline Web Browsing & $\begin{array}{l}\text { Throughput } \\
\text { (kbps) } \\
\text { Latency (ms) }\end{array}$ & 1000 & 1330 & 2660 \\
\hline Video Streaming & $\begin{array}{l}\text { Throughput } \\
\text { (kbps) }\end{array}$ & 1200 & 523 & 261.5 \\
\hline VoIP & $\begin{array}{l}\text { Throughput } \\
\text { (kbps) } \\
\text { Latency (ms) }\end{array}$ & 1200 & 1500 & 5000 \\
\hline
\end{tabular}

(Mean Opinion Score) [26], which can evaluate the QoE providing the QoE to QoS mapping that can be handled and controlled by the system. In the proposed multi-RAT architecture, the functionality of the QoE module provides the following functionalities:

1. Acquire QoE requirements in the form of MOS from the applications run by each UE. Since the latter is subjective to UEs, the module utilizes statistical information aggregating the opinions of many users for each type of service/application.

2. Determine the minimum QoS requirement needed to achieve the desired QoE requirement, as provided by the statistical measurements.

3. Send UE QoS requirement to the Joint RRM function.

For the proposed SpA solution, we assume that a minimum data rate must be guaranteed for the HSPA UEs and will drive the SpA function. A few studies, like the one in [27], define the QoS requirement thresholds to achieve certain MOS values. Table 2 shows a mapping of the considered MOS values that translate to medium, good and excellent QoE (3, 4 and 5 respectively). The basic QoS parameters displayed are throughput and latency that impose different restrictions depending on the type of service used by the UE.

\section{Simulation Results}

The simulation results presented below aim to highlight the effectiveness of the SpA function in the case of an average data rate performance of $800 \mathrm{Kbps}$ is desired for HSPA users. In Fig. 4 the total required bandwidth to support such data rate is depicted for cases where the users experience low $(5 \mathrm{~dB})$, medium $(10 \mathrm{~dB})$ and high $(15 \mathrm{~dB})$ SNRs. The users' SNRs are randomly set by Gaussian processes with mean values of 5,10 and $15 \mathrm{dBs}$ for each respective case. It is evident that as the number of users in the system increases, so does the required bandwidth to support adequate performance. In addition, the higher the average user SNR the less bandwidth is required to support the same number of users. The bandwidth ranges from $10 \mathrm{MHz}$ (the minimum assigned HSPA bandwidth) to $30 \mathrm{MHz}$ in the case where the shared band is 


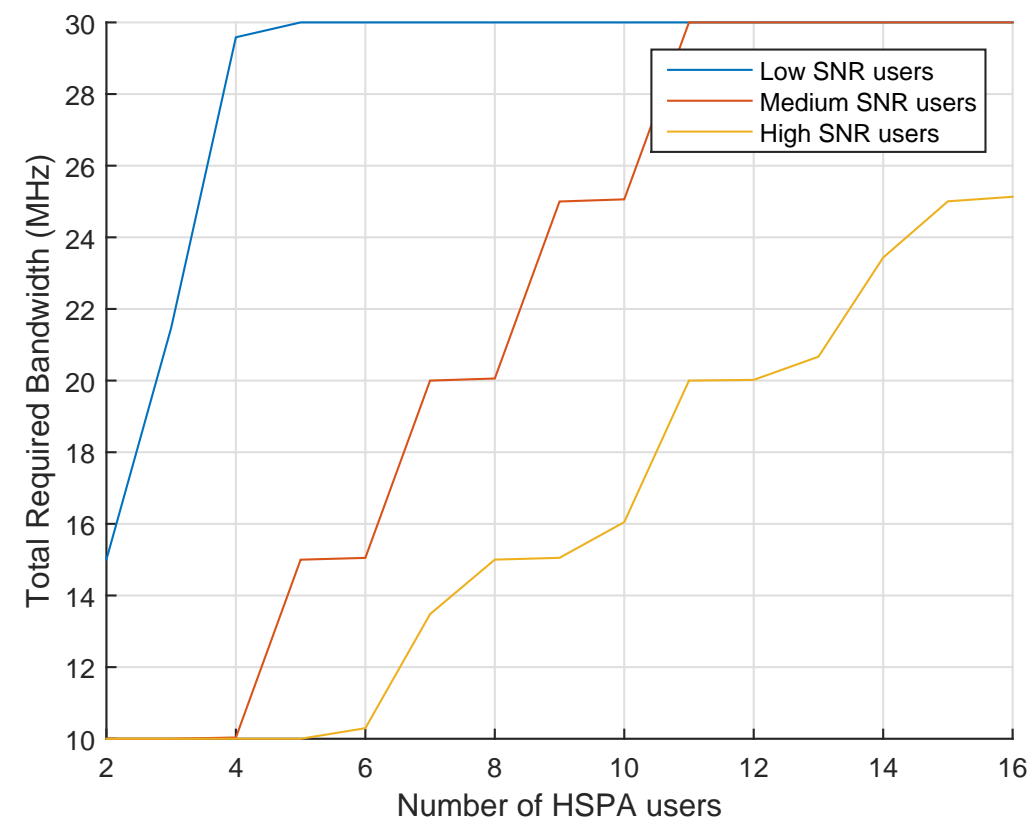

Fig. 4 Total Required BW over number of HSPA users for SpA implementation.

assigned entirely to HSPA, utilizing CA. Note that in the high SNR case, even with 16 HSPA users there is a leftover chunk of the $20 \mathrm{MHz}$ band that can be exploited with LTE-A CA.

In parallel with Fig. 4, Fig. 5 presents the average data rate experienced by the HSPA users. Observe, that when for a specific number of HSPA users we get an increase in bandwidth in Fig. 4, as a result of the application of SpA function $f_{1}$, there is a corresponding increase to the data rate in Fig. 5 due to the application of CA. Note that the average data rate is above the threshold in cases where not the entire shared band is assigned to HSPA as a result of $f_{1}$. If the shared band is assigned to HSPA due to HSPA system load, there is no guarantee about the average data rate performance and thus the average data rate drops below the required threshold, especially in the low SNR case.

The application of $f_{1}$ impacts the LTE-A system as well. The bandwidth of the shared band that is not assigned to HSPA is now exploitable by LTE-A by aggregating with the original $20 \mathrm{MHz}$ carrier. Fig. 6 displays the average LTE-A user data rate in high SNR conditions $(15 \mathrm{~dB})$ for a number of possible bandwidth configurations for LTE-A after the application of the SpA function. The LTE-A system can either utilize from a single $20 \mathrm{MHz}$ carrier if the shared band is assigned to HSPA to a $40 \mathrm{MHz}$ bandwidth as a result of CA. The increase in the average data rate is noteworthy, even for a large number of LTE-A users. 


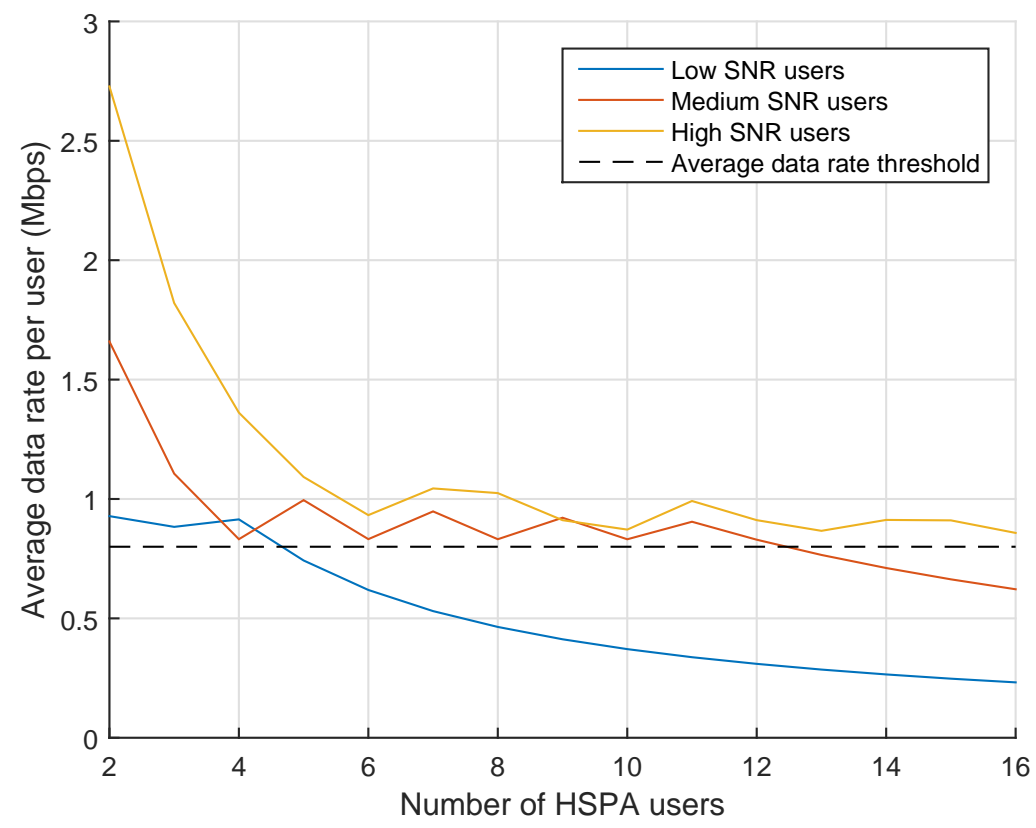

Fig. 5 Average data rate over number of HSPA users for SpA implementation.

In the following simulation results, we aim to demonstrate the effect of SpA and joint SpARA on both HSPA and LTE-A RATs and compare the two methods in terms of throughput performance per RAT. In Fig. 7 the HSPA user throughput in Mbps is plotted for a number of HSPA/LTE-A users that vary from 2 to 16 each. It is evident that joint SpARA outperforms SpA for every combination of HSPA/LTE-A users. Especially for a low number of HSPA/LTE-A users the benefit is significant due to the low average data rate threshold set by the SpA function. Moreover, we can notice that the joint SpARA functions provides a smoother degradation of performance as both RATs number of users increases. With SpA however, HSPA throughput is unaffected by the increase of LTE-A users and only decreases with the number of HSPA users. This is because SpA considers only HSPA load to allocate spectrum based on a target HSPA rate threshold and resource allocation is handled independently by each RAT.

Similar conclusions are made for LTE-A user throughput as shown in Fig. 8. Notice the SpARA surface of Fig. 8 that is always above the respective SpA surface, meaning higher throughput for any number of HSPA/LTE-A users. Again here for a small number of HSPA/LTE-A users the performance is maximized (upper left corner of Fig. 8), while as both HSPA and LTE-A users increase in number, the maximization in throughput is lower. In contrast with Fig. 7, it can be seen that the difference in performance between SpA 


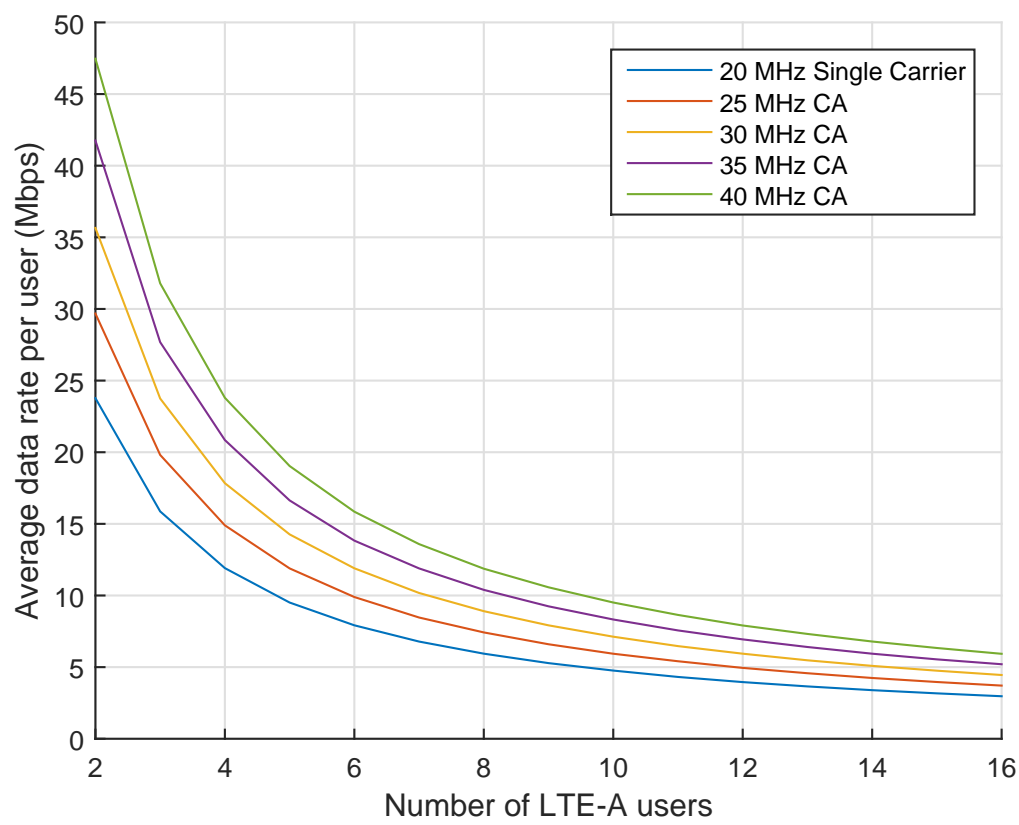

Fig. 6 Average data rate per user for LTE-A possible bandwidth configurations.

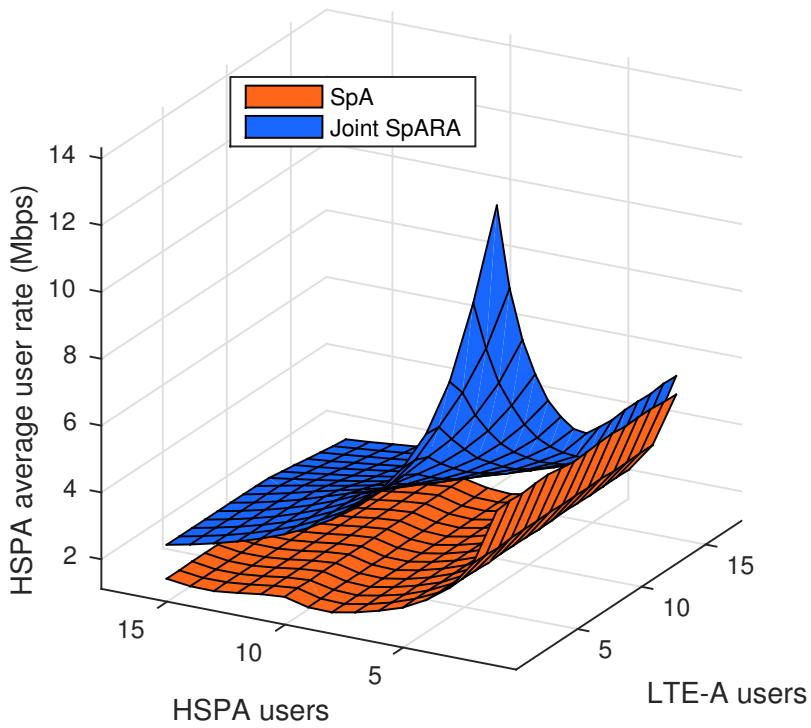

Fig. 7 HSPA user throughput versus number of HSPA/LTE-A users. 


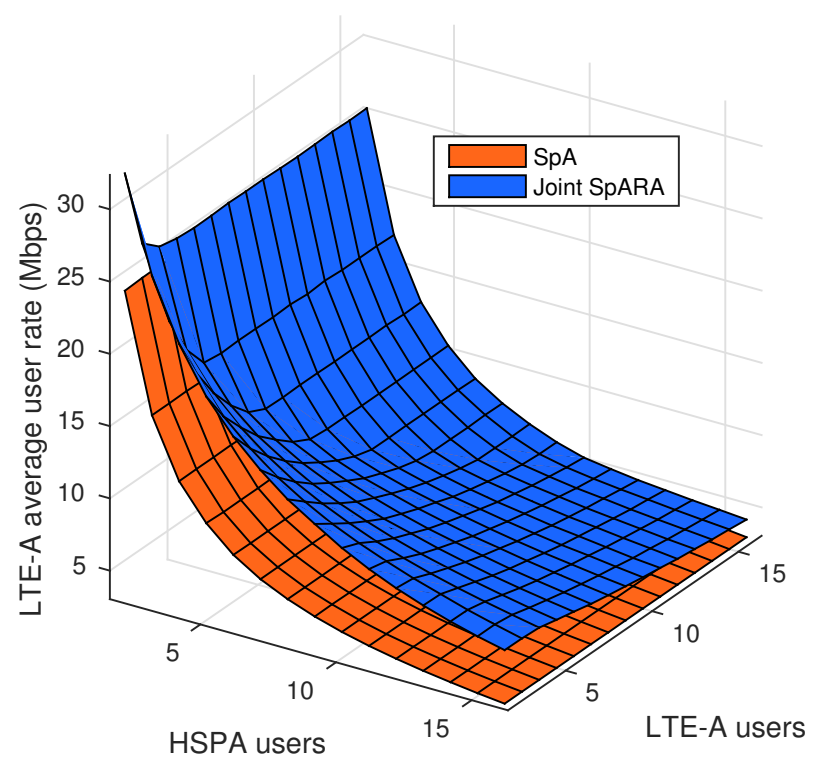

Fig. 8 LTE-A user throughput versus number of HSPA/LTE-A users.

and joint SpARA is not that high, especially for many HSPA/LTE-A user cases. This is due to the data rate threshold set by SpA again. This threshold determines the amount of spectrum that will be spared for LTE-A utilization. This directly affects not only HSPA performance but also LTE-A for the SpA function. Decreasing or increasing the threshold can result in SpA and joint SpARA surfaces in Fig. 7 to diverge or converge and in Fig. 8 to converge or diverge respectively. With that said, in cases where high average data rates are required by the HSPA network, a SpA solution might be a better choice in order to satisfy HSPA users but LTE-A users will not experience as much performance improvement.

Spectrum Utilization: Another goal of the multi-RAT architecture is to utilize the available spectrum more efficiently among the different RATs. To this end, we define a Spectrum Utility (SpU) metric for each RAT and compare the sum spectrum utility for both RATs before and after each SR scheme is applied. The $\mathrm{SpU}$ is defined as the ratio of the average user data rate over the bandwidth exploited by the specific RAT measured in bits/sec/Hz. SpU increases as the average data rate increases and/or the bandwidth decreases so that the system becomes more spectrally efficient. The goal is to prove that the proposed multi-RAT architecture with SpA and joint SpARA functions maximize the sum $\mathrm{SpU}$ of both RATs compared to the initial spectrum assignment situation. Concerning spectrum utilization, the effect of SpA and joint SpARA is demonstrated in Fig. 9 where the sum SpU for both HSPA and LTE-A systems is plotted versus the users SNR values. The topology for 


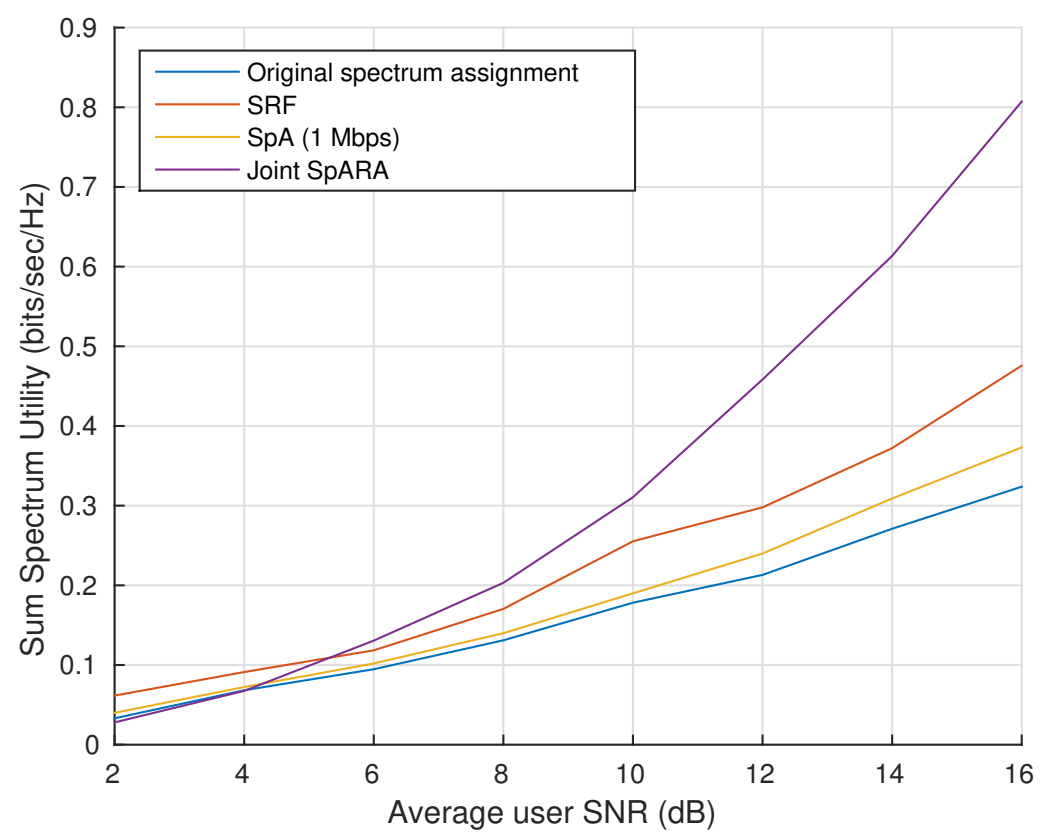

Fig. 9 LTE-A user throughput versus number of HSPA/LTE-A users.

the particular result consists of 10 HSPA plus 10 LTE-A users for the comparison between the original spectrum assignment scheme with fixed bandwidth allocation for each RAT and the proposed SpA and joint SpARA functions.

As can be seen in Fig. 9, the SpU increases as the average user SNR increases, since the users experience higher data rates. As SNRs are higher, the advantages of SpA and joint SpARA compared to the original spectrum assignment situation (30 MHz for HSPA and $20 \mathrm{MHz}$ for LTE-A) are evident. For SpA, the difference might seem insignificant roughly speaking, even around $10 \mathrm{~dB}$; however, since the difference of the sum SpU with the original scheme keeps increasing, the result is noteworthy eventually. Furthermore, with the agile spectrum utilization offered by the joint SpARA function, sum SpU is increased by almost $160 \%$ for $16 \mathrm{~dB}$ SNR compared to the original scheme. Finally, we provide the results with the SRF approach, which achieves a performance higher than $\mathrm{SpA}$; however, this is a static spectrum assignment with more bandwidth assigned to the more spectrally efficient LTE-A. For the same reason, it demonstrates higher SpU that Joint SpARA in the low SNR regime, since Joint SpARA most likely assigns more spectrum to the less spectrally efficient HSPA. This difference however, is quickly diminished and Joint SPARA exponentially increases its SpU to outperform SRF, as SNR increases. 


\section{Conclusion}

This article has argued for multi-RAT aggregation through spectrum reallocation in multi-RAT net-works as a proposal to future wireless networks. We defined a particular management architecture supporting aggregation among multi-RAT spectrum bands. It has noted that with increasing fragmentation of spectrum, and the different characteristics of spectrum and networks that will serve future communication systems realizing the $5 \mathrm{G}$ requirements, advanced integrated solutions will need to be able to combine and reuse the fragmented spectrum providing dynamic opportunities in order to realize the capacity and other benefits of $5 \mathrm{G}$ system.

\section{Acknowledgment}

This work has been supported by the "Spectrum Overlay through Aggregation of Heterogeneous Dispersed Bands" project, ICT-SOLDER, www.ictsolder.eu, FP7 grant agreement number 619687.

\section{References}

\section{References}

1. F. Kaltenberger, F. Foukalas, O. Holland, S. Pietrzyk, S. Thao, G. Vivier, Spectrum overlay through aggregation of heterogeneous dispersed bands, in: Networks and Communications (EuCNC), 2014 European Conference on, 2014, pp. 1-5. doi:10.1109/EuCNC.2014.6882686.

2. O. Galinina, A. Pyattaev, S. Andreev, M. Dohler, Y. Koucheryavy, 5g multirat lte-wifi ultra-dense small cells: Performance dynamics, architecture, and trends, IEEE Journal on Selected Areas in Communications 33 (6) (2015) 1224-1240. doi:10.1109/JSAC.2015.2417016.

3. D. Vujic, J. Certic, UMTS RAN Capacity Analysis for Special Events, Springer Publishing, 2014, pp. 1935-1958.

4. 3GPP, TR 37.870 TSG RAN, Study on multiple radio access technology (multi-rat) joint coordination (release 13), Rel.13 v13.0.0 (June 2015).

5. Next Generation Mobile Networks, RAN Evolution Project, Multi-rat joint radio operation, Rel.13 v 1.1 (March 2015).

6. B. Bangerter, S. Talwar, R. Arefi, K. Stewart, Networks and devices for the $5 \mathrm{~g}$ era, IEEE Communications Magazine 52 (2) (2014) 90-96. doi:10.1109/MCOM.2014.6736748.

7. T. Wang, G. Li, B. Huang, Q. Miao, J. Fang, P. Li, H. Tan, W. Li, J. Ding, J. Li, Y. Wang, Spectrum Analysis and Regulations for 5G, Springer International Publishing, 2017, pp. 27-50.

8. M. Massaro, Next generation of radio spectrum management: Licensed shared access for 5g, Telecommunications Policy 41 (5) (2017) $422-433$.

9. V. Sridhar, T. Casey, H. Hmminen, Flexible spectrum management for mobile broadband services: How does it vary across advanced and emerging markets?, Telecommunications Policy 37 (2) (2013) 178 - 191.

10. S. Han, Y. Liang, B. Soong, S. Li, Dynamic broadband spectrum refarming for ofdma cellular systems, IEEE Transactions on Wireless Communications 15 (9) (2016) 62036214. 
11. A. Alsohaily, E. Sousa, Dynamic spectrum management in multi-radio access technology (rat) cellular systems, Wireless Communications Letters, IEEE 3 (3) (2014) 249-252. doi:10.1109/WCL.2014.022314.130796.

12. A. L. Ramaboli, O. E. Falowo, A. H. Chan, Bandwidth aggregation in heterogeneous wireless networks: A survey of current approaches and issues, Journal of Network and Computer Applications $35 \quad$ (6) (2012) 1674 - 1690. doi:http://dx.doi.org/10.1016/j.jnca.2012.05.015.

URL http://www.sciencedirect.com/science/article/pii/S1084804512001452

13. X. Lin, H. Viswanathan, Dynamic spectrum refarming with overlay for legacy devices, CoRR abs/1302.0320. URL http://arxiv.org/abs/1302.0320

14. Z. Guohua, D. Tianle, Y. Li, A dynamic spectrum re-allocation scheme in gsm and lte co-existed networks, in: Wireless Personal Multimedia Communications (WPMC), 2014 International Symposium on, 2014, pp. 595-600. doi:10.1109/WPMC.2014.7014887.

15. G. Lim, C. Xiong, L. Cimini, G. Li, Energy-efficient resource allocation for ofdma-based multi-rat networks, Wireless Communications, IEEE Transactions on 13 (5) (2014) 2696-2705. doi:10.1109/TWC.2014.032014.131410.

16. N. Abbas, H. Hajj, Z. Dawy, K. Jahed, S. Sharafeddine, An optimized approach to video traffic splitting in heterogeneous wireless networks with energy and qoe considerations, Journal of Network and Computer Applications 83 (2017) $72-88$ doi:http://dx.doi.org/10.1016/j.jnca.2017.01.008.

URL http://www.sciencedirect.com/science/article/pii/S1084804517300140

17. S. Han, Y. chang Liang, B.-H. Soong, Spectrum refarming: A new paradigm of spectrum sharing for cellular networks, in: Global Communications Conference (GLOBECOM), 2014 IEEE, 2014, pp. 893-898. doi:10.1109/GLOCOM.2014.7036922.

18. S. Han, Y.-C. Liang, B.-H. Soong, Robust joint resource allocation for ofdma-cdma spectrum refarming system, Communications, IEEE Transactions on PP (99) (2016) 1-1. doi:10.1109/TCOMM.2016.2517148.

19. D. Das, D. Das, Radio access technology selection in sdn controlled reconfigurable base station, Computers \& Electrical Engineering 61 (2017) 189 - 198 doi:https://doi.org/10.1016/j.compeleceng.2017.04.008.

URL https://www.sciencedirect.com/science/article/pii/S0045790617308595

20. X. Wu, Q. Du, Utility-function-based radio-access-technology selection for heterogeneous wireless networks, Computers \& Electrical Engineering 52 (2016) 171 - 182. doi:https://doi.org/10.1016/j.compeleceng.2015.06.010. URL https://www.sciencedirect.com/science/article/pii/S0045790615002141

21. A. Alsohaily, E. Sousa, Unified radio access network operation for multi-radio access technology cellular systems, in: Telecommunications (ICT), 2014 21st International Conference on, 2014, pp. 32-36. doi:10.1109/ICT.2014.6845075.

22. N. Vucevic, J. Perez-Romero, O. Sallent, R. Agusti, Joint radio resource management for lte-umts coexistence scenarios, in: Personal, Indoor and Mobile Radio Communications, 2009 IEEE 20th International Symposium on, 2009, pp. 12-16. doi:10.1109/PIMRC.2009.5450181.

23. Wireless World Research Forum, Multi-rat network architecture, Tech. Rep. v 2.0 (November 2013).

24. 4G Americas, Hspa+lte carrier aggregation, Tech. rep. (June 2012)

25. M. Nasimi, M. Kousha, F. Hashim, Qoe-oriented cross-layer downlink scheduling for heterogeneous traffics in lte networks, in: 2013 IEEE 11th Malaysia International Conference on Communications (MICC), 2013, pp. 292-297. doi:10.1109/MICC.2013.6805842.

26. K. Lin, W. Wang, X. Wang, W. Ji, J. Wan, Qoe-driven spectrum assignment for $5 \mathrm{~g}$ wireless networks using sdr, IEEE Wireless Communications 22 (6) (2015) 48-55. doi:10.1109/MWC.2015.7368824.

27. A. Aroussi, A. Mellouk, Survey on machine learning-based qoe-qos correlation models, IEEE ComManTel'14 PP (1) (2014) 48-55. doi:10.1109/ComManTel.2014.6825604.

28. P. Casas, A. D'Alconzo, F. Wamser, M. Seufert, B. Gardlo, A. Schwind, P. TranGiaand, R. Schatz, Predicting qoe in cellular networks using machine learning and in-smartphone measurements, IEEE QoMEX'17 PP (1) (2017) 48-55. doi:10.1109/QoMEX.2017.7965687. 
29. 3GPP TS 48.058, Base station controller base tranceiver station (bsc-bts) interface, Release 13 v13.1.0 (April 2016).

30. M. Carpin, A. Zanella, J. Rasool, K. Mahmood, O. Grøndalen, O. N. Østerbø, Scheduling policies for the LTE downlink channel: A performance comparison, CoRR abs/1409.8633.

URL http://arxiv.org/abs/1409.8633 